\title{
Association of antipsychotic use with raised eosinophil count
}

\author{
KONSTANTINOS TSAMAKIS ${ }^{1,2}$, CHRISTOPH MUELLER ${ }^{2,3}$, IOANNIS HORTIS ${ }^{1}$, \\ MARIA KALLERGI $^{4}$, IOANNIS TOLOS ${ }^{1}$, EVANGELOS ALEVYZAKIS ${ }^{1}$, NIKOLAOS SIAFAKAS ${ }^{5}$, \\ ANDREAS OURANIDIS ${ }^{6}$, DIMITRIOS TSIPTSIOS ${ }^{7}$, STYLIANOS KYMPOUROPOULOS ${ }^{1}$, \\ DEMETRIOS A. SPANDIDOS ${ }^{8}$, NIKOLAOS SMYRNIS ${ }^{1}$ and EMMANOUIL RIZOS ${ }^{1}$
}

${ }^{1}$ Second Department of Psychiatry, University General Hospital 'Attikon', National and Kapodistrian University of Athens, Athens 12462, Greece; ${ }^{2}$ Institute of Psychiatry, Psychology and Neuroscience,

King's College London, London SE5 8AF; ${ }^{3}$ South London and Maudsley NHS Foundation Trust, London SE5 8AZ, UK; ${ }^{4}$ Department of Biomedical Engineering, University of West Attica, Campus I, Athens 12243;

${ }^{5}$ Second Laboratory of Microbiology, University General Hospital 'ATTIKON', National and Kapodistrian University of Athens, Medical School, Athens 12462; ${ }^{6}$ Department of Pharmaceutical Technology, School of Pharmacy, Aristotle University of Thessaloniki, Thessaloniki 54636, Greece; ${ }^{7}$ Department of Neurophysiology, South Tyneside and Sunderland NHS Foundation Trust, Sunderland SR47TP, UK;

${ }^{8}$ Laboratory of Clinical Virology, School of Medicine, University of Crete, Heraklion 71003, Greece

Received November 12, 2020; Accepted January 26, 2021

DOI: $10.3892 /$ etm.2021.9944

\begin{abstract}
The current study aimed to assess the possibility of an association between first and second generation antipsychotic medication and raised eosinophil count. A total of 22 in-patients at the psychiatric unit of the University General Hospital 'Attikon', a tertiary hospital, were included in the present study. Patients had received antipsychotic monotherapy and did not have any co-morbidities or require additional treatments. Patients were monitored weekly and their eosinophil count was assessed. One-way ANOVA and summary measures analysis were applied to study the effect of time and medication type on the absolute eosinophil concentration (or relative percentage) for each patient. The differences in mean eosinophil concentrations or relative percentage by patient and time were also assessed. An increase in the absolute concentration and the relative percentage of eosinophils over time was observed in patients receiving Olanzapine, Haloperidol and Aripiprazole. However, there was no difference between individual medications. In conclusion, antipsychotics may be associated with increased eosinophil count over time; however, larger studies involving
\end{abstract}

Correspondence to: Dr Konstantinos Tsamakis, Second Department of Psychiatry, University General Hospital 'Attikon', National and Kapodistrian University of Athens, Rimini 1, Athens 12462, Greece

E-mail:ktsamakis@gmail.com

Key words: antipsychotics, eosinophil count, monotherapy, first and second generation, olanzapine, haloperidol, aripiprazole more patients and a longer follow-up are required to reach a definitive conclusion.

\section{Introduction}

Eosinophils are a type of white blood cell formed from stem cells in the bone marrow. Raised eosinophil count is associated with a wide variety of allergic, rheumatologic, infectious, neoplastic and rare idiopathic disorders (1). Several drugs, including antimalarials, non-steroid anti-inflammatories and anticonvulsants, are associated with eosinophilia (2).

Eosinophilia has been documented as a well-established side effect of the antipsychotic drug clozapine, as it is seen in $\sim 1 \%$ of clozapine-treated patients (3). However, limited data are available regarding the potential effect of other antipsychotic medications on eosinophil count $(4,5)$.

Despite the lack of systematic studies, eosinophilia related to antipsychotic use has been described in a number of case reports; olanzapine, most notably, has been associated with increased eosinophil count (6-8) whilst it has also been found to cause eosinophilic pleural effusion (6), drug reaction with eosinophilia and systemic symptoms (DRESS) syndrome (9), eosinophilic myocarditis (10), as well as hypersensitivity syndrome (11). Aripiprazole has also been associated with DRESS syndrome (12) and eosinophilic myocarditis (13), Quetiapine has been associated with eosinophilia $(14,15)$, refractory cardiac myocarditis and DRESS syndrome (14), and one case report described risperidone-induced acute eosinophilic pneumonia (16).

The purpose of this study was to generate a larger case series on the possible association between raised eosinophil count and any of the following six -first and second-generation antipsychotic drugs: Olanzapine, Aripiprazole, Quetiapine, Risperidone, Haloperidol and Amisulpride. The present study 
did not include any patients on Clozapine, since its relation to increased eosinophil count is well documented in the literature $(3,17)$.

\section{Materials and methods}

Ethics approval. The study was conducted at the Department of Psychiatry at the University General Hospital 'Attikon', a tertiary care multispecialty hospital in Athens, Greece. Ethics approval for this study and approval for use of patient data were obtained from the Scientific Committee of 'Attikon' Hospital (Athens, Greece) with the approval number (of the research project of which this present study is a part) being 14/210109. All patients gave informed consent on admission with regards to the use of their data, which they could withdraw at any point. All procedures performed in the study were in accordance with the ethical standards of the Helsinki Declaration (1964) and its later amendments.

Data collection and inclusion criteria. Data were obtained from the database of electronic and paper patient records of the Department of Psychiatry of Attikon Hospital. Patients admitted to the Department of Psychiatry between April 4, 2016 and November 1, 2019 who received standard clinical treatment at the time of the study were included if they met the following criteria: i) Sufficient duration of hospitalisation ( $>2$ weeks), so that the fluctuation in the eosinophil count could be monitored; ii) monotherapy treatment with one antipsychotic medication in-hospital; iii) no administration of other medication that could contribute to eosinophil count fluctuations (e.g., antidepressants or antibiotics) during hospital stay; iv) access to a reliable past medical history, including electronic medical records; v) either no history of receiving antipsychotics for a 6-month period prior to hospitalization or being antipsychotic naïve; vi) if they had at least two blood tests measuring eosinophil count during their hospital stay; and vii) no history of any serious organic illness (such as respiratory problems, including pneumonia, intestinal problems, such as colitis, skin conditions, recent or chronic infections, allergies, cancer, autoimmune disorders and haematological disorders) that could in any way affect eosinophil count. Also, no history of any specific eosinophilic disorders, such as eosinophilic granulomatosis with polyangiitis (EGPA, formerly known as Churg-Strauss), eosinophilic fasciitis, eosinophilic lung disorders, hypereosinophilic syndrome and eosinophilic leukemia. The medical history was obtained through the patients' own self-report and also through the available electronic medical records of the patients, which were accessible once they were admitted to hospital (Fig. 1, for details and flow-chart).

Statistical analysis. Patients included were tested every week for a period of 2-6 weeks and the levels of eosinophils (absolute eosinophil count in $\mathrm{K} / \mu \mathrm{l}$ and eosinophil WBC percentage) were extracted. The first measurement of the eosinophils represents the baseline eosinophil count before the patients started treatment with antipsychotic medication.

To study the effect of time and drug type on the concentration (or relative \%) the slopes of the least square regression lines for each patient are presented and were analysed with single factor ANOVA.

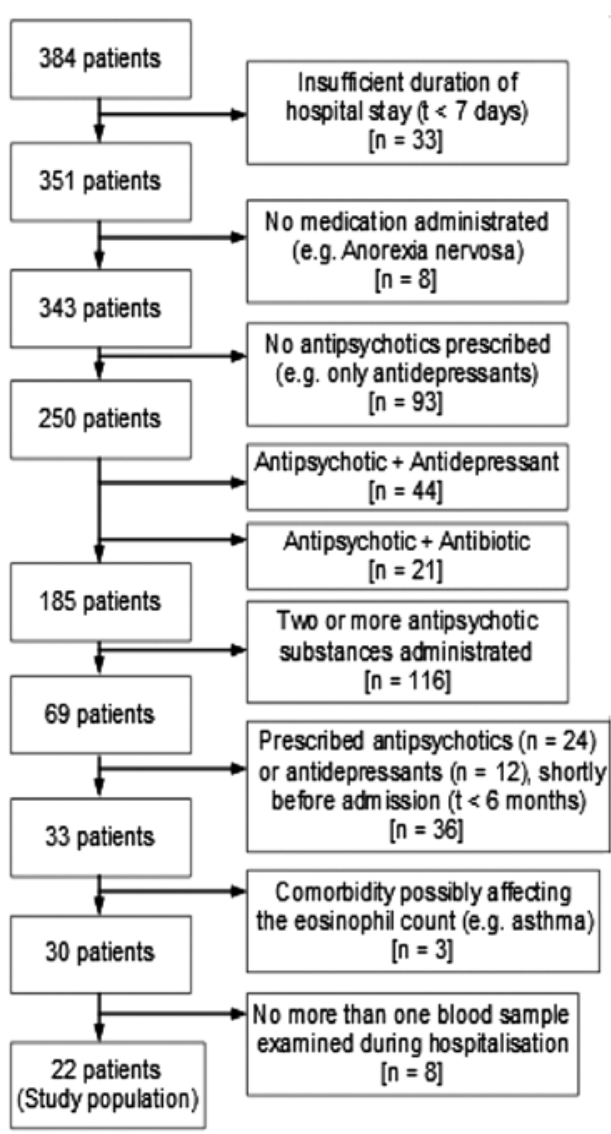

Figure 1. Flowchart of patient selection.

Summary measures analysis and single factor (or one way) ANOVA were applied to test the difference in the means of the eosinophil concentrations or relative $\%$ when there were sufficient $(3$ or more) patients in a group by patient and by time $(18,19)$.

\section{Results}

Sample characteristics. A total of 384 patients admitted in the $31 / 2$-year period and receiving antipsychotic treatment were identified. Of these, 22 patients met the inclusion criteria of the present study (Fig. 1)

The mean age of the 22 patients included was 45.2 years, $36 \%$ were female, and the average duration of the hospital stay was 22 days. Of those, 9 (40.9\%) received monotherapy with Olanzapine, 6 (27.3\%) Haloperidol, 3 (13.6\%) Aripiprazole, 2 (9.1\%) Amisulpride, 1 (4.5\%) Quetiapine and 1 (4.5\%) Risperidone.

Changes in eosinophil count associated with antipsychotic use. Different patients responded differently to each drug in terms of eosinophil count. Figs. 2A and B, 3A and B, and $4 \mathrm{~A}$ and $\mathrm{B}$ show the corresponding changes in the absolute count (Figs. 2A, 3A and 4A) and relative \% (Figs. 2B, 3B and 4B) for Olanzapine, Haloperidol and Aripiprazole, respectively (where three or more patients were included for each drug) over time. A trendline was fitted to the data of each patient and is shown in the figures. Increases in the mean absolute count and relative $\%$ were observed for all antipsychotic medications. Haloperidol showed the greatest increase overall, 
$\mathbf{A}$
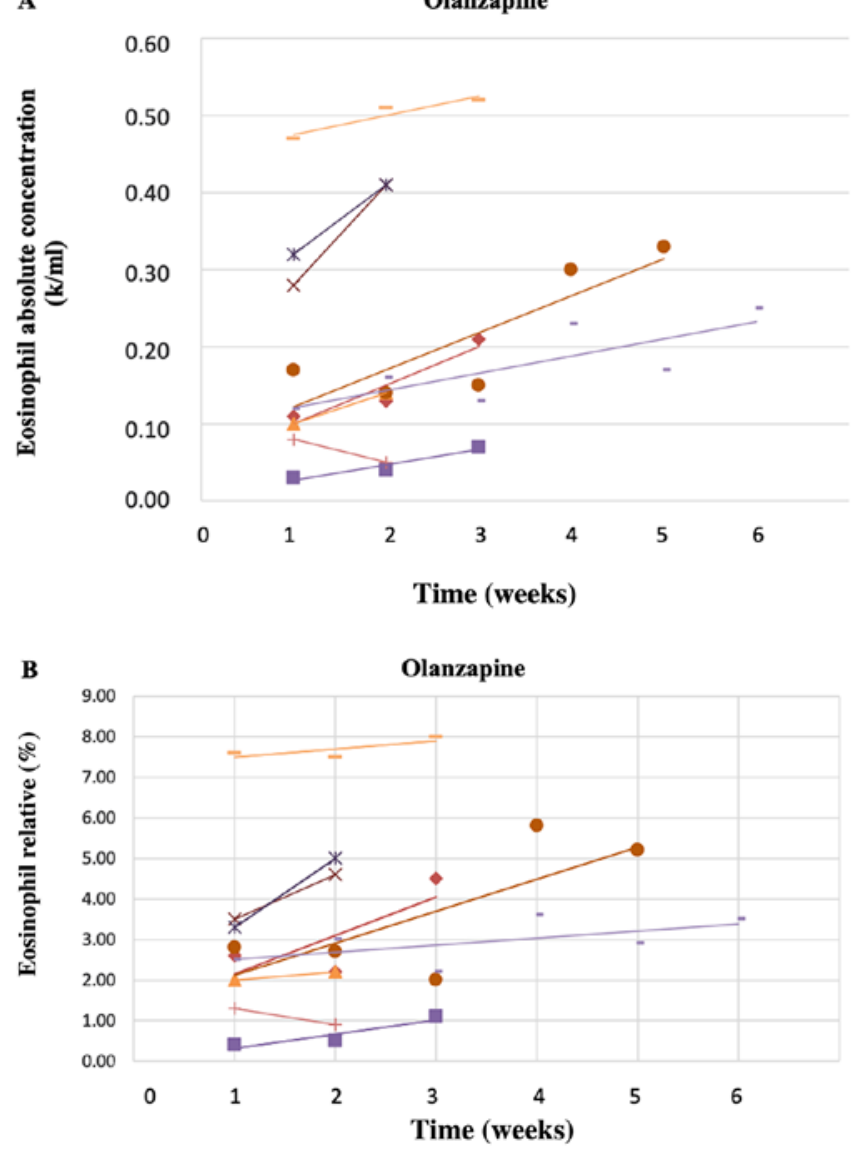

Figure 2. Eosinophil (A) absolute concentration $(\mathrm{K} / \mu \mathrm{l})$ and (B) relative percentage over time for 9 patients receiving Olanzapine. Each coloured line represents one individual patient. Each symbol indicates one eosinophil measurement (the same symbol is used for the different measurements of the same patient).

$\sim 100 \%$ for both the absolute count and the $\%$. Of note, the antipsychotic doses used were the regular -within therapeutic range- doses that are used in clinical practice. More specifically, for Olanzapine the doses used were between 5 and $20 \mathrm{mg}$ daily, for Aripiprazole they were between 10 and $30 \mathrm{mg}$ daily and for Haloperidol they were between 5 and $20 \mathrm{mg}$ daily.

Significant differences for eosinophils' relative $\%$ over time were detected for 9 patients in the Olanzapine group $(\mathrm{F}=12.3>\mathrm{F}$-critical; $\mathrm{P}<0.001)$, the 6 patients in the Haloperidol group $(\mathrm{F}=5.8>\mathrm{F}$-critical; $\mathrm{P}=0.006)$, and the 3 patients in the Aripiprazole group $(\mathrm{F}=11.3>\mathrm{F}$-critical; $\mathrm{P}=0.009)$, while significant differences were also seen in the absolute eosinophil values in patients on Olanzapine $(\mathrm{F}=17.2>\mathrm{F}$-critical; $\mathrm{P}<0.001)$, Haloperidol $(\mathrm{F}=8.1>\mathrm{F}$-critical, $\mathrm{P}=0.002)$ and Aripiprazole $(\mathrm{F}=12.0>\mathrm{F}$-critical; $\mathrm{P}=0.008)$.

No statistically significant differences in the means among drugs over time and no differences in the absolute and relative $\%$ eosinophil count from one week to the next were detected for the three antipsychotics Olanzapine $(\mathrm{F}=0.3 ; \mathrm{P}=0.91)$, Haloperidol $(\mathrm{F}=0.7 ; \mathrm{P}=0.59)$ and Aripiprazole $(\mathrm{F}=0.1 ; \mathrm{P}=0.97)$.

\section{Discussion}

The present study investigated the impact of antipsychotic monotherapy on the eosinophil count of a case series of
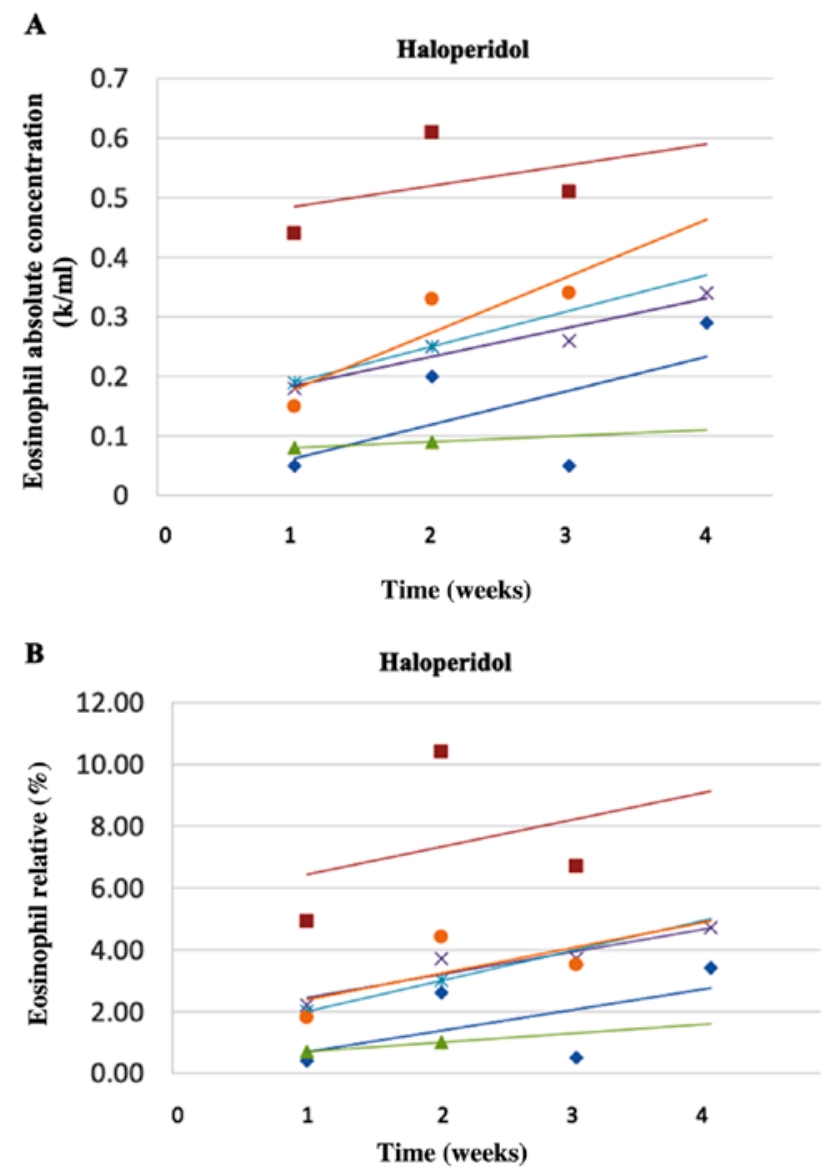

Figure 3. Eosinophil (A) absolute concentration $(\mathrm{K} / \mu \mathrm{l})$ and (B) relative percentage over time for 6 patients receiving Haloperidol. Each coloured line represents one individual patient. Each symbol indicates one eosinophil measurement (the same symbol is used for the different measurements of the same patient).

22 psychiatric inpatients over time. The present findings suggest a linear increase in the absolute concentration and the relative $\%$ of eosinophils with time for at least 3 of the antipsychotic drugs, Olanzapine, Haloperidol and Aripiprazole (for which there were at least 3 patients on each). An increase between the first and last measurement of the eosinophil count was also seen for the other three antipsychotics (Amisulpride, Risperidone and Quetiapine) which were taken by groups of two patients or a single patient (data not shown).

Although there were differences in the increase of the eosinophil count among patients taking the same medication as a function of time, there was no difference in the rate of increase between medications, which means each medication caused a similar effect on the eosinophils (increases with similar variance) of the patients with time.

To the best of our knowledge, this is the first case series investigating the association between antipsychotics and eosinophilic count. The study's strengths include that patients were on antipsychotic monotherapy and that both first and second generation antipsychotics were investigated. In addition, strict exclusion criteria were applied to minimize the influence of other factors that could cause eosinophilia. This strict approach has generated a more homogenous, but small case series lacking statistical power to detect significant findings between medications. Hence, our findings indicate 

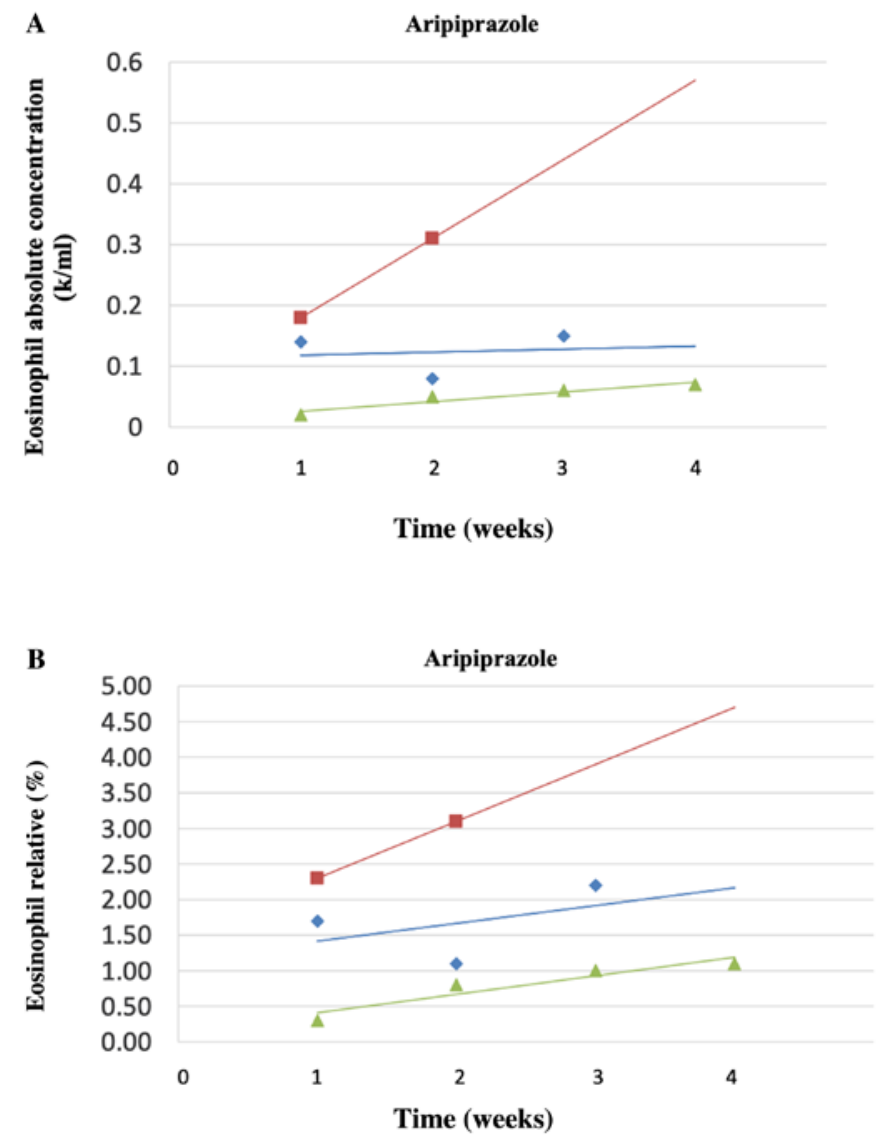

Figure 4. Eosinophil (A) absolute concentration $(\mathrm{K} / \mu \mathrm{l})$ and (B) relative percentage over time for 3 patients receiving Aripiprazole. Each coloured line represents one individual patient. Each symbol indicates one eosinophil measurement (the same symbol is used for the different measurements of the same patient).

that antipsychotic medications have an effect on the eosinophil count, but more patients need to be studied for a longer period of time. Also, since the primary aim of the study was to explore the effect of antipsychotic use on eosinophil count, data on neutrophils were not collected and analysed; however, this matter will be dealt with in a future study.

Although there are no confirmed mechanisms by which antipsychotics may cause eosinophilia, two possible explanations can be found in the literature. The first mechanism is called pharmacologic interaction with immune receptors (p-I concept) and suggests that medications may be associated with eosinophilia by activating T-cells. This would lead to the release of cytokines stimulating eosinophils directly and/or eosinophil precursors (20). The second possible mechanism concerns the molecule of serotonin. According to animal studies, serotonin and eotaxin have been demonstrated to have an eosinophil chemoattractant profile. This most likely occurs via the 5HT2A receptor (21), which has been reported to be a mediator of inflammation outside of the central nervous system $(21,22)$. The serotoninergic action of antipsychotic drugs is mediated via their affinity for 5-HT2A receptors, especially at low dosage. It is therefore arguable that the serotonergic action of antipsychotic drugs through antagonism of 5-HT2A receptors could result in a rise in eosinophil count. Serotonin eosinophilic-specific chemo-attracting action induced by a low dose antipsychotic (risperidone) has been previously implicated as a mechanism leading to eosinophilic pneumonia (16).

In conclusion, the present case series shows a possible signal in the association between antipsychotic use and raised eosinophil count. The small sample size however precludes inferring causal associations. However, it adds to the literature in an under-researched area, and suggests that potential serious consequences of raised eosinophils, such as DRESS syndrome (9), eosinophilic myocarditis (13) and pneumonia (16), warrant further investigations. Our estimation is that a sufficiently powered future study should have at least 8-10 patients per drug, and 5-8 consecutive measurements (i.e., follow-up for 5-8 weeks) per patient for all patients. Such a study is being constructed, since data collection regarding eosinophils and antipsychotic use remains ongoing in our department, and the results will be presented in the near future once a sufficiently powered sample is reached.

\section{Acknowledgements}

Not applicable.

\section{Funding}

No funding was received.

\section{Availability of data and materials}

The datasets used and/or analyzed during the current study are available from the corresponding author on reasonable request.

\section{Authors' contributions}

KT and CM wrote the original draft, edited and critically revised the manuscript. KT and $\mathrm{IH}$ confirmed the authenticity of all the raw data. KT, CM, MK, IT, NSi, SK, AO, EA, ER, DT, DAS and NSm made substantial contributions to the conception and design of the current study and interpreted the data. IH, IT, EA and ER acquired the data. MK, CM, IH and KT made substantial contributions to data handling, figure preparation and analysis of the data. All authors critically revised and edited the manuscript. All authors substantially contributed to the conception, writing and revision of the work and read and approved the final content of the manuscript.

\section{Ethics approval and consent to participate}

The authors assert that all procedures contributing to this work comply with the ethical standards of the relevant national and institutional committees on human experimentation and with the Helsinki Declaration of 1975, as revised in 2008. The study was conducted at the Department of Psychiatry at the University General Hospital 'Attikon', a tertiary care multispecialty hospital in Athens, Greece. Ethics Approval for this study and approval for use of patient data were obtained by the Scientific Committee of 'Attikon' Hospital (Athens, Greece) (approval no. 14/210109). All patients provided informed consent on admission with regards to the use of their data, which they could withdraw at any time. 


\section{Patient consent for publication}

Not applicable.

\section{Competing interests}

DAS is the Editor-in-Chief for the journal, but had no personal involvement in the reviewing process, or any influence in terms of adjudicating on the final decision, for this article. The other authors declare that they have no competing interests.

\section{References}

1. Klion AD: Eosinophilia: A pragmatic approach to diagnosis and treatment. Hematology Am Soc Hematol Educ Program, 92-97: 2015.

2. Rauscher $\mathrm{C}$ and Freeman A: Drug-induced eosinophilia. Allergy Asthma Proc 39: 252-256, 2018

3. Kadiyala PK, Ahmed MA, Pinto DA and Mathai JP: Clozapine induced eosinophilia: An often neglected important adverse effect. Indian J Psychiatry 57: 429-430, 2015.

4. Gentile S: Safety concerns associated with second-generation antipsychotic long-acting injection treatment. A systematic update. Horm Mol Biol Clin Investig 36, 2018.

5. Rettenbacher MA,Hofer A, KemmlerG and FleischhackerWW: Neutropenia induced by second generation antipsychotics: A prospective investigation. Pharmacopsychiatry 43: 41-44, 2010.

6. Huang J, Yu Y, Lin W, Zhang D, Deng Z and Ding Q Olanzapine-induced peripheral eosinophilia and eosinophilic pleural effusion: A case report. Medicine (Baltimore) 97: e9996, 2018.

7. Tournikioti K, Douzenis A, Antoniadou A, Papazahos K, Moschos C, Papageorgiou C and Rizos EN: Eosinophilia Associated With Olanzapine. J Clin Psychopharmacol 36: 180-181, 2016.

8. Mathias S, Schaaf LWN and Sonntag A: Eosinophilia associated with olanzapine. J Clin Psychiatry 63: 246-247, 2002.

9. Penchilaiya V, Kuppili PP, Preeti K and Bharadwaj B: DRESS syndrome: Addressing the drug hypersensitivity syndrome on combination of Sodium Valproate and Olanzapine. Asian J Psychiatr 28: 175-176, 2017.
10. Vang T, Rosenzweig M, Bruhn CH, Polcwiartek C, Kanters JK and Nielsen J: Eosinophilic myocarditis during treatment with olanzapine-report of two possible cases. BMC Psychiatry 16: 70, 2016.

11. Raz A, Eilam O, Hayek T, Bergman R and Yungerman T: A Case report of olanzapine-induced hypersensitivity syndrome. Am J Med Sci 321: 156-158, 2001.

12. Taleb S, Zgueb Y, Ouali U, Jomli R, Kort Y and Nacef F: Drug reaction with eosinophilia and systemic symptoms syndrome related to aripiprazole therapy. J Clin Psychopharmacol 39: 691-693, 2019.

13. Christoffersen RK, Vestergård LD, Høimark L and Vesterby A: Eosinophilic myocarditis and sudden unexpected death in a younger patient treated with antipsychotics. Ugeskr Laeger 173: 2799-2800, 2011 (In Danish).

14. Hagiwara H, Fukushima A, Iwano H and Anzai T: Refractory cardiac myocarditis associated with drug rash with eosinophilia and systemic symptoms syndrome due to anti-bipolar disorder drugs: A case report. Eur Hear J Case Rep 2: yty100, 2018.

15. Chen L, Tan P and Tan X: Case report of eosinophilia induced by quetiapine. Shanghai Arch psychiatry 27: 374-377, 2015.

16. Rizos E, Tsigkaropoulou E, Lambrou P, Kanakaki M, Chaniotou A, Alevyzakis E and Liappas I: Risperidone-induced acute eosinophilic pneumonia. In Vivo 27: 651-653, 2013.

17. Lee J, Takeuchi H, Fervaha G, Powell V, Bhaloo A, Bies R and Remington G: The effect of clozapine on hematological indices: A 1-Year Follow-Up Study. J Clin Psychopharmacol 35: 510-516, 2015.

18. Matthews JN, Altman DG, Campbell MJ and Royston P: Analysis of serial measurements in medical research. BMJ 300: 230-235, 1990

19. Matthews JNS: A refinement to the analysis of serial data using summary measures. Stat Med 12: 27-37, 1993.

20. Pichler WJ, Beeler A, Keller M, Lerch M, Posadas S, Schmid D, Spanou Z, Zawodniak A and Gerber B: Pharmacological interaction of drugs with immune receptors: The p-i concept. Allergol Int 55: 17-25, 2006.

21. Boehme SA, Lio FM, Sikora L, Pandit TS, Lavrador K, Rao SP and Sriramarao P: Cutting edge: Serotonin is a chemotactic factor for eosinophils and functions additively with eotaxin. J Immunol 173: 3599-3603, 2004.

22. Baganz NL and Blakely RD: A Dialogue between the immune system and brain, spoken in the language of serotonin. ACS Chem Neurosci 4: 48-63, 2013.

This work is licensed under a Creative Commons Attribution-NonCommercial-NoDerivatives 4.0 International (CC BY-NC-ND 4.0) License. 